\title{
Erratum to: Continuous production of biohythane from hydrothermal liquefied cornstalk biomass via two-stage high-rate anaerobic reactors
}

Bu-Chun $\mathrm{Si}^{1}$, Jia-Ming Li ${ }^{1}$, Zhang-Bing Zhu' ${ }^{1}$ Yuan-Hui Zhang ${ }^{2}$, Jian-Wen Lu', Rui-Xia Shen ${ }^{1}$, Chong Zhang ${ }^{3,4}$, Xin-Hui Xing ${ }^{3,4}$ and Zhidan Liu ${ }^{1 *}$

\section{Erratum to: Biotechnol Biofuels (2016) 9:254 DOI 10.1186/s13068-016-0666-z}

After this article [1] was published, the authors realised that the funding number for The National Natural Science Foundation of China was incorrect. The correct funding number is as follows: 51561145013, U1562107.

\section{Author details}

${ }^{1}$ Laboratory of Environment-Enhancing Energy (E2E), and Key Laboratory of Agricultural Engineering in Structure and Environment, Ministry of Agriculture, College of Water Resources and Civil Engineering, China Agricultural University, Beijing 100083, China. ${ }^{2}$ Department of Agricultural and Biological Engineering, University of Illinois at Urbana-Champaign, Urbana 61801, USA.

${ }^{3}$ Key Laboratory of Industrial Biocatalysis of Ministry of Education of China, Beijing 100084, China. ${ }^{4}$ Department of Chemical Engineering, Institute of Biochemical Engineering, Tsinghua University, Beijing 100084, China.
The online version of the original article can be found under doi:10.1186/s13068-016-0666-z.

Received: 19 January 2017 Accepted: 24 January 2017

Published online: 03 February 2017

Reference

1. Si B-C, Li J-M, Zhu Z-B, Zhang Y-H, Lu J-W, Shen R-X, Zhang C, Xing X-H, Liu Z. Continuous production of biohythane from hydrothermal liquefied cornstalk biomass via two-stage high-rate anaerobic reactors. Biotechnol Biofuels. 2016;9:254. doi:10.1186/s13068-016-0666-z.
*Correspondence: zdliu@cau.edu.cn

${ }^{1}$ Laboratory of Environment-Enhancing Energy (E2E), and Key Laboratory of Agricultural Engineering in Structure and Environment, Ministry of Agriculture, College of Water Resources and Civil Engineering, China Agricultural University, Beijing 100083, China

Full list of author information is available at the end of the article 